\title{
Big size is not all, even better!
}

\author{
Roberto Ivan Troisi ${ }^{1,2}$, Mariano Cesare Giglio ${ }^{1}$, Roberto Montalti ${ }^{3}$ \\ ${ }^{1}$ Department of Clinical Medicine and Surgery, Federico II University of Naples, Naples, Italy; ${ }^{2}$ Department of Liver and Small Bowel \\ Transplantation, Hepato-Biliary and Pancreatic Surgery, King Faisal Specialist Hospital and Research Center, Riyadh, Kingdom of Saudi Arabia; \\ ${ }^{3}$ Department of Public Health, Federico II University of Naples, Naples, Italy \\ Correspondence to: Roberto Ivan Troisi, MD. Department of Clinical Medicine and Surgery, Federico II University Naples, Via Pansini 5, 80131, \\ Naples, Italy. Email: roberto.troisi@unina.it. \\ Comment on: Cloyd JM, Mizuno T, Kawaguchi Y, et al. Comprehensive Complication Index Validates Improved Outcomes Over Time Despite \\ Increased Complexity in 3707 Consecutive Hepatectomies. Ann Surg 2018. [Epub ahead of print].
}

Submitted Mar 26, 2019. Accepted for publication Apr 10, 2019.

doi: 10.21037/hbsn.2019.04.08

View this article at: http://dx.doi.org/10.21037/hbsn.2019.04.08

We read with interest the article entitled "Comprehensive Complication Index validates improved outcomes over time despite increased complexity in 3707 consecutive hepatectomies" by Cloyd et al. (1). This article focused on the correlation between surgical volume and the development of competency in open liver surgery. The clinical results of completing a substantial number of hepatic procedures determines the increase in the complex procedures (i.e., major or two-stage hepatectomy, or repeat hepatectomy) and a significant decrease in the overall complication and mortality rates, which combine to a reduce surgical time, intraoperative bleeding, and length of hospital stay. The multidisciplinary approach to a single clinical case as an example to follow was critical in improving the clinical results, as demonstrated in other studies, is also well highlighted. The take-home message of this study is how important it is for complex liver surgery to be performed by experienced surgeons at high-volume centres.

Because this article discusses the importance of appropriate methodologies (or surgical techniques) that are applied in liver surgery to improve overall results, we believe that there are some limitations besides those that were highlighted in the Discussion section.

In our opinion, the authors fail to demonstrate how the experience gained through conventional surgery could introduce and facilitate mini-invasive approaches (laparoscopic or robotic), which are both performed with increasing frequency, especially for minor resections. Different types of liver resections are currently performed using minimally invasive techniques, mostly at experienced centres, except for complex vascular and biliary reconstructions. Interventions that were formerly contraindicated using a laparoscopic approach can be performed with robotic assistance, facilitating reconstruction phases.

The article reports that the proportion of complex operations over time (i.e., major hepatectomies) has increased, reflecting proficiency. However, others, instead, consider the significant reduction in major hepatectomies to be a sign of good practice, which indicates mastery of the hepatic surgical anatomy (2). Parenchymal sparing surgery with respect of segmental inflow, outflow, and biliary drainage of a single segment or hepatic sector using the vein-preserving approach would be preferable because it leads to a higher percentage of future liver remnant, thereby reducing blood loss, overall complications, and postoperative liver failure $(2,3)$. We know that the degree of complexity should be preferably indicated by a difficulty score because it is much more representative of the true difficulty of a liver resection (4). For example, resection of the antero-medial sector (SV-VIII) involving the resection of just two segments is much more difficult than the left lateral sectionectomy, which also consists in the removal of two adjacent segments.

Another important aspect that was not considered by the authors is the learning curve of each individual surgeon. Thus, it is not known how many surgeries (and which type) are necessary to perform before moving on to the more complex operations for conventional and minimally invasive surgery. The literature lacks guidelines on the learning curve for open surgery, while it is advised that a "mandatory" stepwise approach be combined with formal training in 
laparoscopic liver surgery to decrease the impact of the learning curve in the early stages and obtain credentials to be considered a "safe and proficient" minimally invasive liver surgeon (with related health-care reimbursement issues) (5).

Another aspect that was not considered in the article is the importance of the knowledge and role of intraoperative ultrasound in guiding liver surgery. The liver surgeon should have appropriate knowledge of this method because it allows planning of surgical strategies, correctly visualizing tumoral lesions aiming at R0 resections, and performing parenchyma-saving resections to potentially minimise intraoperative bleeding (6).

Moreover, this article does not address specific types of interventions such as Associating Liver Partitioning and portal vein Ligation for Staged hepatectomy (ALPPS) procedures, liver transplantation, and living donors hepatectomies. Thus, we believe that experience in complex liver surgery is a prerequisite for successfully performing liver transplantations, especially partial liver transplantations.

Additionally, the role and the importance of the Enhanced Recovery After Surgery (ERAS) protocols is not sufficiently described. Fast-track protocols, if properly used in open and in minimally invasive surgery, allow a significant reduction in the postoperative hospital stay, time to functional recovery, and overall complication rates (7).

This article provided us the opportunity to highlight the most important aspects of modern liver surgery, which strives to improve the quality and excellence for referrals of the most complex cases at high-volume centres, multidisciplinary case discussion, mandatory use of intraoperative ultrasound, and eventually the implementation of the ERAS protocols. Adequate training and surgical progression are required for actual new technological challenges that are offered by the minimally invasive approaches, real time navigation, and $3 \mathrm{D}$ printing.

Cite this article as: Troisi RI, Giglio MC, Montalti R. Big size is not all, even better! HepatoBiliary Surg Nutr 2019;8(5):539540. doi: 10.21037/hbsn.2019.04.08

\section{Acknowledgments}

None.

\section{Footnote}

Conflicts of Interest: The authors have no conflicts of interest to declare.

\section{References}

1. Cloyd JM, Mizuno T, Kawaguchi Y, et al. Comprehensive Complication Index Validates Improved Outcomes Over Time Despite Increased Complexity in 3707 Consecutive Hepatectomies. Ann Surg 2018. [Epub ahead of print].

2. Kingham TP, Correa-Gallego C, D'Angelica MI, et al. Hepatic parenchymal preservation surgery: decreasing morbidity and mortality rates in 4,152 resections for malignancy. J Am Coll Surg 2015;220:471-9.

3. Tomassini F, Bonadio I, Smeets P, et al. Safety analysis of the oncological outcome after vein-preserving surgery for colorectal liver metastases detached from the main hepatic veins. Langenbecks Arch Surg 2015;400:683-91.

4. Lee MK, Gao F, Strasberg SM. Perceived complexity of various liver resections: results of a survey of experts with development of a complexity score and classification. J Am Coll Surg 2015;220:64-9.

5. Wakabayashi G, Cherqui D, Geller DA, et al.

Recommendations for laparoscopic liver resection: a report from the second international consensus conference held in Morioka. Ann Surg 2015;261:619-29.

6. Torzilli G, Procopio F. State of the Art of Intraoperative Ultrasound in Liver Surgery: Current Use for Resectionguidance. Chirurgia (Bucur) 2017;112:320-5.

7. Li L, Chen J, Liu Z, et al. Enhanced recovery program versus traditional care after hepatectomy: A meta-analysis. Medicine (Baltimore) 2017;96:e8052. 